\title{
Enfermedad de von Willebrand como manifestación clínica inhabitual del hipotiroidismo primario. Caso clínico
}

\author{
Rodrigo Concha $\mathrm{N}^{\mathrm{a}}$, María Alejandra Borzone $\mathrm{V}^{\mathrm{a}}$, Marcelo \\ Castillo $\mathbf{N}^{1}$, Alberto Rossle $\mathrm{S}^{2}$, Iván Q uevedo $\mathrm{L}^{3}$. \\ Acquired von Willebrand disease as \\ an unusual manifestation of primary \\ hypothyroidism. Report of two cases
}

Patients with hypothyroidism may have a minor increase in their bleeding tendency, causing easy bruising and menorrhagia. There is a positive correlation between factor VIII coagulant activity and thyroxin serum levels. Thus, patients with hypothyroidism have an acquired coagulation defect that is reversible with thyroxin supplementation. We report two sisters, aged 13 and 11 years, who met the criteria for von Willebrand's disease at the age of eight. Both sisters had a primary hypothyroidism due to Hashimoto's thyroiditis, diagnosed three years later. Thyroid hormone replacement normalized von Willebrand factor, factor VIII, and the bleeding diathesis disappeared. Acquired von Willebrand's disease is an unusual manifestation of hypothyroidism. However the possibility of hypothyroidism should be considered in patients presenting with von Willebrand disease (Rev Méd Chile 2005; 133: 813-6).

(Key Words: Hashimoto disease; Thyroiditis, autoimmune; von Willebrand disease)

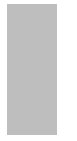

Recibido el 22 de diciembre, 2004. Aceptado el 25 de abril, 2005.

Secciones de ${ }^{2}$ Hematología y ${ }^{3}$ Endocrinología, Departamento de Medicina Interna, ${ }^{1}$ Departamento de Especialidades Médicas, Facultad de Medicina, Universidad de Concepción. aAlumno de la carrera de medicina, Facultad de Medicina, Universidad de Concepción.

$\mathrm{E}^{1}$ hipotiroidismo primario es una de las patologías crónicas más frecuentes de la población adulta. Estudios epidemiológicos muestran que en Chile la prevalencia del hipotiroidismo corresponde a $7 \% 1$, cifra algo superior a las comunicadas en

Correspondencia a: Dr. Iván Quevedo l. Avenida Andalué $\mathrm{N}^{\circ}$ 1825; San Pedro de la Paz, Concepción-Chile. E mail: equevedo@udec.cl otros lugares del mundo, donde la prevalencia oscila entre 3 y $6 \% 2,3$. Los casos avanzados de hipotiroidismo primario no ofrecen mayor dificultad diagnosticada, dado que los síntomas y signos dan cuenta de una historia de cansancio fácil, astenia, bradipsiquia e intolerancia al frío. El paciente refiere presentar constipación, aumento de peso y dolores osteomusculares inespecíficos, y en las mujeres puede agregarse hipermenorrea o amenorrea. En el examen físico, llamará la atención la facie vultuosa, palidez variable de la piel, cejas ralas, cabello grueso y voz grave ${ }^{4}$. Sin 
embargo, los pacientes con hipotiroidismo primario, en ocasiones debutan con una variada gama de presentaciones inhabituales, las que pueden abarcar diferentes sistemas y aparatos del cuerpo. La importancia de reconocer las formas inhabituales de presentación del hipotiroidismo, radica en que el diagnóstico tardío puede tener consecuencias graves $\mathrm{y}$, por otro lado, la sustitución con levotiroxina puede prevenir completamente el cuadro clínico ${ }^{5,6}$.

En el hipotiroidismo primario se han descrito manifestaciones inhabituales tales como musculares (miopatías) ${ }^{7}$, cardiovasculares (derrame pericárdico, cardiomegalia, isquemia miocárdica silente) $^{8,9}$, reumatológicas (hiperuricemia) ${ }^{10}$, psiquiátricas (depresión) ${ }^{11}$ y hematológicas. Entre las manifestaciones hematológicas, destacan la anemia (normocítica hipocrómica por disminución del consumo de oxígeno y macrocítica por déficit de vitamina B12) ${ }^{12,13}$ y una mayor tendencia a los sangrados con traumatismos menores. Se han comunicado casos de hemorragias graves en pacientes con mixedema hipotiroideo ${ }^{14}$, en los cuales los niveles plasmáticos del factor de von Willebrand (FvW) son menos de la mitad en los pacientes hipotiroideos que en las personas normales, y los niveles plasmáticos del FvW se normalizan con la sustitución con levotiroxina revirtiendo completamente el cuadro clínico ${ }^{14-16}$.

Presentamos los casos de dos hermanas con diagnóstico de enfermedad de von Willebrand (EvW), a quienes se les detectó un hipotiroidismo primario por tiroiditis crónica y cuyas manifestaciones hemorrágicas desaparecieron con la sustitución con levotiroxina.

\section{CASO 1}

Paciente de 13 años, sexo femenino, con antecedentes de otitis crónica, sin antecedentes familiares de hemopatías y asintomática hasta la edad de 8 años, cuando comenzó con epistaxis frecuentes y sangrado digestivo bajo, en al menos tres ocasiones. A esa misma edad y dado los antecedentes de diatesis hemorrágica, se le realizó estudio hematológico en evaluación preoperatoria de amigdalectomía. Se diagnosticó una EvW basado en TTPA de 48,2 s (VR: 25-39) factor VIII: $\mathrm{C}$ de 50\% (VR 57-122), FvW: Ag de 37\% (VR: 50-146) y cofactor de ristocetina de 37\% (VR: 50-172). Previo y durante a la amigdalectomía, se le indicó crioprecipitado, con lo que la paciente evolucionó sin complicaciones. En los años posteriores la paciente persistió con epistaxis a repetición.

A los 10 años de edad consultó por aumento de volumen cervical anterior indoloro. El estudio hormonal mostró TSH mayor a 10 en dos ocasiones (VR: 0,5-5,0) y anticuerpos antiperoxidasa positivos, diagnosticándose un hipotiroidismo primario por tiroiditis crónica de Hashimoto. Se comenzó tratamiento con levotimoxina $100 \mathrm{ug} /$ día, logrando normalizar los niveles plasmáticos de hormonas timoideas al mes de tratamiento. Desde el inicio de la terapia de sustitución con levotiroxina, la paciente no ha presentado ningún episodio de epistaxis o sangrado digestivo, incluso tuvo su menarquia a los 12 años. Actualmente sus ciclos son regulares $\mathrm{IV} / 28$, sin coágulos, TTPA: 32,3 s, factor VIII: C de 80\% y FvW: Ag de 95\%.

\section{CASO 2}

Paciente de 11 años, sexo femenino, con antecedentes de otitis crónica, asintomática hasta los 8 años cuando comenzó con epistaxis a repetición. Por el hecho de tener una hermana con EvW se le realizó un estudio hematológico previo a cirugía de extirpación de amígdalas, que mostró hallazgos compatibles con una EvW: TTPA de 42,5 s (VR: 25-39) y con FvW de 27\% (VR: 50-146). Previo y durante la cirugía se le indicó ácido tranexámico y crioprecipitado, evolucionando sin incidentes. En los años posteriores la paciente persistió con epistaxis a repetición.

A los 10 años, dado antecedentes de hermana con hipotiroidismo primario, se le realizó estudio endocrinológico que mostró que ella también era portadora de un hipotiroidismo primario por tiroiditis crónica de Hashimoto. Actualmente, la paciente está en tratamiento con levotiroixina 100 ug/día, desde el inicio de la terapia de sustitución hormonal no ha vuelto a presentar epistaxis, su TTPA es de 35 s y el FvW de $86 \%$.

\section{DisCUSIÓN}

La EvW es la hematopatía hereditaria más común, estimándose que afecta a una persona entre 800 a 
1.00017. El FvW es una proteína multimétrica y heterogénea, que posee dos funciones principales: 1) facilita la adherencia plaquetaria en un medio de grandes tensiones tangenciales, al formar un puente entre los receptores de membrana de la plaqueta y el subendotelio vascular, 2) sirve como proteína transportadora del factor VIII. Una disminución pequeña en la concentración del factor en el plasma conlleva la pérdida selectiva de multímeros de alto peso molecular, disminuyendo de esta forma la adherencia plaquetaria con la consecuente aparición de hemorragia ${ }^{18}$.

La EvW puede ser clínicamente heterogénea, pero existen algunos signos clínicos que son comunes a todos los síndromes. En los casos leves, la hemorragia surge sólo después de una cirugía o traumatismo, pero en los pacientes con un ataque más grave éstos sufren epistaxis o hemorragia espontánea de mucosas de la boca, tubo gastrointestinal o genitourinarias. El patrón diagnóstico más común es la combinación de: 1) la prolongación del tiempo de sangría 2) disminución en la concentración plasmática del FvW 3) disminución paralela en la actividad del cofactor de ristocetina y 4) menor actividad del factor VIII ${ }^{19}$. En nuestro caso ambas hermanas tuvieron manifestaciones clínicas y de laboratorio que certificaron el diagnóstico de EvW. Sin embargo, por otro lado ninguna de las hermanas evidenció las manifestaciones clásicas del hipotiroidismo, ni tampoco los hallazgos esperables en un niño con hipotiroidismo como antecedente de talla baja, retardo de la edad ósea, aumento del vello corporal, síndrome edematoso o disminución del rendimiento escolar ${ }^{20}$.

La forma en que se relacionan ambas enfermedades parece ser que durante la evolución del hipotiroidismo se produce una disminución en la

\section{REFERENCIAS}

1. Fardelia C, Poggi H, Gloger G, Rojas A, Velásquez C, Barroileth S et al. Alta prevalencia de enfermedad tiroidea en sujetos aparentemente sanos que concurren a control de salud. Rev Méd Chile 2001; 129: $155-60$.

2. Vanderpump MP, Tunbridge WM, French JM, Appleton D, Bates D. Clark F et al. The incidence of síntesis de proteínas del FvW por ausencia o disminución de tiroxinas, y con la normalización del déficit de hormona tiroidea se produce una normalización del FvW. Los mecanismos a través de los cuales se revertiría las manifestaciones clínicas de la EvW serían: 1) una liberación aumentada del FvW desde las células endoteliales dada por una mayor susceptibilidad a la epinefrina post terapia, 2) una estimulación no específica de la síntesis de proteínas hepáticas por acción de la tiroxina ${ }^{21,22}$.

Dado que ambas hermanas desde el inicio del tratamiento de reemplazo con hormonas tiroideas no han presentado episodios de epistaxis, hemorragias, ni equimosis que representen una actividad o manifestación de la EvW, y más aún, la mayor de las hermanas ya presenta menstruaciones normales en el último año, además de existir una normalización de los parámetros hematológicos se puede sostener que existe una normalización de la actividad hemostática de ambas pacientes.

El hecho de que la EvW sea una de las enfermedades hematológicas más frecuentes en Chile, con una prevalencia de $0,8 \% 23$, y que el hipotiroidismo primario sea a su vez una patología con alta prevalencia en la población general, hace pensar que existe un número significativo de pacientes con EvW que no presentan esta enfermedad desde el nacimiento, sino que ésta se deba a una aparición más tardía dentro del cuadro de un hipotiroidismo primario que no ha presentado las manifestaciones clínicas clásicas. Esto último nos hace plantear que la búsqueda de la asociación entre ambas enfermedades podría implicar un mejor pronóstico de la enfermedad hematológica, al ser necesario sólo la sustitución con levotiroxina para revertir en casos similares a los presentados las manifestaciones hemorrágicas.

thyroid disorders in the community: A twenty years follow-up of the Whickham Survey. Clin Endocrinol 1995; 43: 55-68.

3. Arem R, Escalante D. Subclinical hypothyroidism: epidemiology, diagnosis and significance. Arch Intern Med 1996; 41: 213-50.

4. Bastiene PA, Bonnyns M, VAN Haelst L Natural history of primary myxedema. Am J Med 1985; 79: 91-100. 
5. Michaud P. Formas inhabituales de presentación del hipotiroidismo primario. Rev Méd Chile 1987; 115: 872-80.

6. KieIN I, Levey GS. Unusual manifestations of hypothyroidism. Arch Intern Med 1984; 144: 123-8.

7. Finsterer J, Stoluberger C, Grossegger C, Kroiss A. Hypothyroid myopathy with unusually high serum creatine kinase values. Horm Res 1999; 52: 205-8.

8. Sawka AM, Fatourechi W. Subclinical thyroid dysfunction and the heart. Ann Intern Med 2003; 139: 866-7.

9. Quevedo I, Mosso L, Domínguez JM, Fajuri A, Quintana JC. Isquemia miocárdica reversible del hipotiroidismo. Rev Méd Chile 2001; 129: 1320-4.

10. Giordano N, Santacroce C, Mattil G, Geraci S, Amendola A, Gennari C. Hyperuricemia and gout in thyroid endocrine disorders. Clin Exp Rheumatol 2001; 19: 661-5.

11. JACKSON IM. The thyroid axis and depression. Thyroid 1998; 8: 951-6.

12. Das KC, Murkhersee M, Sarkar TJ, Dash RS, Rastogi GK. Erythropoiesis and erythropietin in hypo and hyperthyroidism. J Clin Endocrinol Metab 1975; 40: 211-20.

13. Caplan RH, Davis K, Bengston B, Smith MS. Serum folate and vitamin B12 levels in hypothyroid and hyperthyroid patients. Arch Intern Med 1975; 135: 701-4.

14. Ford HC, CARTer JM. Haemostasis in hypothyroidism. Postgrad Med J 1990; 66: 280-4.

15. Myrup B, Bregengard C, Faber J. Primary haemostasis and thyroid disease. J Int Med 1995; 238: 59-63.
16. Attivissimo LA, Lichtman SM, Kifin I. Acquired von Willebrand's syndrome causing a hemorragic diathesis in a patient with hypothyroidism. Thyroid 1995; 5: 399-401.

17. Rodeghiero F, Castamann G, Dini E. Epidemiological investigation of the prevalence of von Willebrand's disease. Blood 1987; 69: 454-60.

18. Bцоом AL von Willebrand factor: Clinical features of inherited and acquired disorders. Mayo Clin Proc 1991; 66: 743-51.

19. SADLER JE. A revised classification of von Willebrand disease. For the subcommittee on von Willebrand factor of the scientific and standardization committee of the international society on thrombosis and haemostasis. Thromb Haemost 1994; 71: 520-8.

20. Foley TP JR. Hypothyroidism. Pediatr Rev 2004; 25: 94-100.

21. Veyradier A, Jenkins LS, Fressinaud E, Meyer D. Acquired von Willebrand syndrome: From pathophysiology to management. Thromb Haemost 2000; 84: 175-8.

22. Franchini M, De Girancol M, Luppi G, Manzato F, Brazzarola P, Bottura D et al. Efficacy of desmopressin as surgical prophylaxis in patient with acquired von Willebrand disease undergoing thyroid surgery. Haemophilia 2002; 8: 142-4.

23. Quiroga T, Pérez M, Rodríguez J, Muñoz B, Aranda E, Morales M et al. Skin and mucous membrane hemorrhages: clinical assessment, study sequence and relative frequency of hereditary diseases of the hemostasis in a Chilean population. Rev Méd Chile 1997; 125: 409-18.

Agradecimientos

Los autores agradecen la valiosa colaboración en la redacción y ordenamiento de la Sra. Ana Cristina Contreras, Secretaria Centro Saval de Concepción. 